\title{
ESTIMATES FOR MEASURES OF SECTIONS OF CONVEX BODIES
}

\author{
ALEXANDER KOLDOBSKY
}

\begin{abstract}
A $\sqrt{n}$ estimate in the hyperplane problem with arbitrary measures has recently been proved in [K3]. In this note we present analogs of this result for sections of lower dimensions and in the complex case. We deduce these inequalities from stability in comparison problems for different generalizations of intersection bodies.
\end{abstract}

\section{INTRODUCTION}

The following inequality has recently been proved in [K3]. Let $K$ be an origin symmetric convex body in $\mathbb{R}^{n}$, and let $\mu$ be a measure on $K$ with even continuous non-negative density $f$ so that $\mu(B)=\int_{B} f$ for every Borel subset of $K$. Then

$$
\mu(K) \leq \sqrt{n} \frac{n}{n-1} c_{n} \max _{\xi \in S^{n-1}} \mu\left(K \cap \xi^{\perp}\right)|K|^{1 / n},
$$

where $c_{n}=\left|B_{2}^{n}\right|^{\frac{n-1}{n}} /\left|B_{2}^{n-1}\right|<1, B_{2}^{n}$ is the unit Euclidean ball in $\mathbb{R}^{n}$, and $|K|$ stands for volume of proper dimension. Note that $c_{n}<1$ for every $n$.

In the case of volume, when $f=1$ everywhere on $K$, inequality (1) was proved in [MP, p. 96]. Another argument follows from [G, Theorem 8.2.13]; in [G] this argument is attributed to Rolf Schneider. Also, in the case of volume the constant $\sqrt{n}$ can be improved to $C n^{1 / 4}$, where $C$ is an absolute constant, as shown by Klartag [Kl] who removed a logarithmic term from an earlier estimate of Bourgain [Bo3]. These results are much more involved. The question of whether $n^{1 / 4}$ can also be removed in the case of volume is the matter of the hyperplane conjecture [Bo1, Bo2, Ba, MP]; see the book [BGVV] for the current state of the problem.

In this note we prove analogs of inequality (1) for sections of lower dimensions and in the complex case; see Theorems 2 and 4, respectively. As in [K3], the proofs are based on certain stability results for generalizations of intersection bodies. 


\section{LOWER DIMENSIONAL SECTIONS}

We need several definitions and facts. A closed bounded set $K$ in $\mathbb{R}^{n}$ is called a star body if every straight line passing through the origin crosses the boundary of $K$ at exactly two points different from the origin, the origin is an interior point of $K$, and the Minkowski functional of $K$ defined by

$$
\|x\|_{K}=\min \{a \geq 0: x \in a K\}
$$

is a continuous function on $\mathbb{R}^{n}$.

The radial function of a star body $K$ is defined by

$$
\rho_{K}(x)=\|x\|_{K}^{-1}, \quad x \in \mathbb{R}^{n} .
$$

If $x \in S^{n-1}$ then $\rho_{K}(x)$ is the radius of $K$ in the direction of $x$.

If $\mu$ is a measure on $K$ with even continuous density $f$, then

$$
\mu(K)=\int_{K} f(x) d x=\int_{S^{n-1}}\left(\int_{0}^{\|\theta\|_{K}^{-1}} r^{n-1} f(r \theta) d r\right) d \theta
$$

Putting $f=1$, one gets

$$
|K|=\frac{1}{n} \int_{S^{n-1}} \rho_{K}^{n}(\theta) d \theta=\frac{1}{n} \int_{S^{n-1}}\|\theta\|_{K}^{-n} d \theta .
$$

For $1 \leq k \leq n-1$, denote by $G r_{n-k}$ the Grassmanian of $(n-k)$ dimensional subspaces of $\mathbb{R}^{n}$. The $(n-k)$-dimensional spherical Radon transform $R_{n-k}: C\left(S^{n-1}\right) \mapsto C\left(G r_{n-k}\right)$ is a linear operator defined by

$$
R_{n-k} g(H)=\int_{S^{n-1} \cap H} g(x) d x, \quad \forall H \in G r_{n-k}
$$

for every function $g \in C\left(S^{n-1}\right)$.

The polar formulas (2) and (3), applied to sections of $K$, express volume in terms of the spherical Radon transform:

$$
\begin{aligned}
\mu(K \cap H) & =\int_{K \cap H} f=\int_{S^{n-1} \cap H}\left(\int_{0}^{\|\theta\|_{K}^{-1}} r^{n-k-1} f(r \theta) d r\right) d \theta \\
& =R_{n-k}\left(\int_{0}^{\|\cdot\|_{K}^{-1}} r^{n-k-1} f(r \cdot) d r\right)(\xi) .
\end{aligned}
$$

and

$$
|K \cap H|=\frac{1}{n-k} \int_{S^{n-1} \cap \xi^{\perp}}\|\theta\|_{K}^{-n+k} d \theta=\frac{1}{n-k} R_{n-k}\left(\|\cdot\|_{K}^{-n+k}\right)(\xi) .
$$


The class of intersection bodies was introduced by Lutwak [L] and played a crucial role in the solution of the Busemann-Petty problem; see $[\mathrm{G}, \mathrm{K} 1]$ for definition and properties. A more general class of bodies was introduced by Zhang [Z] in connection with the lower dimensional Busemann-Petty problem. Denote

$$
R_{n-k}\left(C\left(S^{n-1}\right)\right)=X \subset C\left(G r_{n-k}\right) .
$$

Let $M^{+}(X)$ be the space of linear positive continuous functionals on $X$, i.e. for every $\nu \in M^{+}(X)$ and non-negative function $f \in X$, we have $\nu(f) \geq 0$.

An origin-symmetric star body $K$ in $\mathbb{R}^{n}$ is called a generalized $k$ intersection body if there exists a functional $\nu \in M^{+}(X)$ so that for every $g \in C\left(S^{n-1}\right)$,

$$
\int_{S^{n-1}}\|x\|_{K}^{-k} g(x) d x=\nu\left(R_{n-k} g\right) .
$$

When $k=1$ we get the class of intersection bodies. It was proved by Grinberg and Zhang [GZ, Lemma 6.1] that every intersection body in $\mathbb{R}^{n}$ is a generalized $k$-intersection body for every $k<n$. More generally, as proved later by Milman [Mi], if $m$ divides $k$, then every generalized $m$-intersection body is a generalized $k$-intersection body.

We need the following stability result for generalized $k$-intersection bodies.

Theorem 1. Suppose that $1 \leq k \leq n-1, K$ is a generalized $k$ intersection body in $\mathbb{R}^{n}, f$ is an even continuous function on $K, f \geq 1$ everywhere on $K$, and $\varepsilon>0$. If

$$
\int_{K \cap H} f \leq|K \cap H|+\varepsilon, \quad \forall H \in G r_{n-k},
$$

then

$$
\int_{K} f \leq|K|+\frac{n}{n-k} c_{n, k}|K|^{k / n} \varepsilon
$$

where $c_{n, k}=\left|B_{2}^{n}\right|^{\frac{n-k}{n}} /\left|B_{2}^{n-k}\right|<1$.

Proof : Use polar formulas (4) and (5) to write the condition (7) in terms of the $(n-k)$-dimensional spherical Radon transform: for all $H \in G r_{n-k}$

$$
R_{n-k}\left(\int_{0}^{\|\cdot\|_{K}^{-1}} r^{n-k-1} f(r \cdot) d r\right)(H) \leq \frac{1}{n-k} R_{n-k}\left(\|\cdot\|_{K}^{-n+k}\right)(H)+\varepsilon .
$$


Let $\nu$ be the functional corresponding to $K$ by (6), apply $\nu$ to both sides of the latter inequality (the direction of the inequality is preserved because $\nu$ is a positive functional) and use (6). We get

$$
\begin{gathered}
\int_{S^{n-1}}\|\theta\|_{K}^{-k}\left(\int_{0}^{\|\theta\|_{K}^{-1}} r^{n-k-1} f(r \theta) d r\right) d \theta \\
\leq \frac{1}{n-k} \int_{S^{n-1}}\|\theta\|_{K}^{-n} d \theta+\varepsilon \nu(1) .
\end{gathered}
$$

Split the integral in the left-hand side into two integrals and then use $f \geq 1$ as follows:

$$
\begin{gathered}
\int_{S^{n-1}}\left(\int_{0}^{\|\theta\|_{K}^{-1}} r^{n-1} f(r \theta) d r\right) d \theta \\
+\int_{S^{n-1}}\left(\int_{0}^{\|\theta\|_{K}^{-1}}\left(\|\theta\|_{K}^{-k}-r^{k}\right) r^{n-k-1} f(r \theta) d r\right) d \theta \\
\geq \int_{K} f+\int_{S^{n-1}}\left(\int_{0}^{\|\theta\|_{K}^{-1}}\left(\|\theta\|_{K}^{-k}-r^{k}\right) r^{n-k_{1}} d r\right) d \theta \\
=\int_{K} f+\frac{1}{n-k}|K| .
\end{gathered}
$$

Now estimate $\nu(1)$ by first writing $1=R_{n-k} 1 /\left|S^{n-k-1}\right|$ and then using definition (6), Hölder's inequality and $\left|S^{n-1}\right|=n\left|B_{2}^{n}\right|$ :

$$
\begin{aligned}
& \nu(1)=\frac{1}{\left|S^{n-k-1}\right|} \nu\left(R_{n-k} 1\right)=\frac{1}{\left|S^{n-k-1}\right|} \int_{S^{n-1}}\|\theta\|_{K}^{-k} d \theta \\
& \leq \frac{1}{\left|S^{n-k-1}\right|}\left|S^{n-1}\right|^{\frac{n-k}{n}}\left(\int_{S^{n-1}}\|\theta\|_{K}^{-n} d \theta\right)^{\frac{k}{n}} \\
& =\frac{\varepsilon}{\left.\left|S^{n-k-1 \mid}\right| S^{n-1}\right|^{\frac{n-k}{n}} n^{k / n}|K|^{k / n}=\frac{n}{n-k} c_{n, k}|K|^{k / n}} .
\end{aligned}
$$

Combining (9), (10) and (11) we get

$$
\int_{K} f+\frac{1}{n-k}|K| \leq \frac{n}{n-k}|K|+\frac{n}{n-k} c_{n, k}|K|^{k / n} \varepsilon
$$


It was proved in $[\mathrm{KM}]$ (generalizing the result for $k=1$ from [K2]) that if $L$ is a generalized $k$-intersection body and $\mu$ is a measure with even continuous density, then

$$
\mu(L) \leq \frac{n}{n-k} c_{n, k} \max _{H \in G r_{n-k}} \mu(L \cap H)|L|^{k / n} .
$$

We show now that it is possible to extend this inequality to arbitrary origin-symmetric convex bodies in $\mathbb{R}^{n}$ at the expense of an extra constant $n^{k / 2}$.

Theorem 2. Suppose that $L$ is an origin-symmetric convex body in $\mathbb{R}^{n}$, and $\mu$ is a measure with even continuous non-negative density $g$ on L. Then

$$
\mu(L) \leq n^{k / 2} \frac{n}{n-k} c_{n, k} \max _{H \in G r_{n-k}} \mu(L \cap H)|L|^{k / n} .
$$

Proof : By John's theorem $[\mathrm{J}]$, there exists an origin-symmetric ellipsoid $K$ such that

$$
\frac{1}{\sqrt{n}} K \subset L \subset K
$$

The ellipsoid $K$ is an intersection body ([G, Corollary 8.1.7]), and every intersection body is a generalized $k$-intersection body for every $k$ ([GZ, Lemma 6.1]). Let $f=\chi_{K}+g \chi_{L}$, where $\chi_{K}, \chi_{L}$ are the indicator functions of $K$ and $L$, then $f \geq 1$ everywhere on $K$. Put

$$
\varepsilon=\max _{H \in G r_{n-k}}\left(\int_{K \cap H} f-|K \cap H|\right)=\max _{H \in G r_{n-k}} \int_{L \cap H} g .
$$

Now we can apply Theorem 1 to $f, K, \varepsilon$ (the function $f$ is not necessarily continuous on $K$, but the result holds by a simple approximation argument). We get

$$
\begin{gathered}
\mu(L)=\int_{L} g=\int_{K} f-|K| \\
\leq \frac{n}{n-k} c_{n, k}|K|^{k / n} \max _{H \in G r_{n-k}} \int_{L \cap H} g \\
\leq n^{k / 2} \frac{n}{n-k} c_{n, k}|L|^{k / n} \max _{H \in G r_{n-k}} \mu(L \cap H),
\end{gathered}
$$

because $K \subset \sqrt{n} L$, so $|K| \leq n^{n / 2}|L|$. 


\section{The COMPLEX CASE}

Origin symmetric convex bodies in $\mathbb{C}^{n}$ are the unit balls of norms on $\mathbb{C}^{n}$. We denote by $\|\cdot\|_{K}$ the norm corresponding to the body $K$ :

$$
K=\left\{z \in \mathbb{C}^{n}:\|z\|_{K} \leq 1\right\} .
$$

In order to define volume, we identify $\mathbb{C}^{n}$ with $\mathbb{R}^{2 n}$ using the standard mapping

$$
\xi=\left(\xi_{1}, \ldots, \xi_{n}\right)=\left(\xi_{11}+i \xi_{12}, \ldots, \xi_{n 1}+i \xi_{n 2}\right) \mapsto\left(\xi_{11}, \xi_{12}, \ldots, \xi_{n 1}, \xi_{n 2}\right) .
$$

Since norms on $\mathbb{C}^{n}$ satisfy the equality

$$
\|\lambda z\|=|\lambda|\|z\|, \quad \forall z \in \mathbb{C}^{n}, \forall \lambda \in \mathbb{C},
$$

origin symmetric complex convex bodies correspond to those origin symmetric convex bodies $K$ in $\mathbb{R}^{2 n}$ that are invariant with respect to any coordinate-wise two-dimensional rotation, namely for each $\theta \in$ $[0,2 \pi]$ and each $\xi=\left(\xi_{11}, \xi_{12}, \ldots, \xi_{n 1}, \xi_{n 2}\right) \in \mathbb{R}^{2 n}$

$$
\|\xi\|_{K}=\left\|R_{\theta}\left(\xi_{11}, \xi_{12}\right), \ldots, R_{\theta}\left(\xi_{n 1}, \xi_{n 2}\right)\right\|_{K},
$$

where $R_{\theta}$ stands for the counterclockwise rotation of $\mathbb{R}^{2}$ by the angle $\theta$ with respect to the origin. We shall say that $K$ is a complex convex body in $\mathbb{R}^{2 n}$ if $K$ is a convex body and satisfies equations (13). Similarly, complex star bodies are $R_{\theta}$-invariant star bodies in $\mathbb{R}^{2 n}$.

For $\xi \in \mathbb{C}^{n},|\xi|=1$, denote by

$$
H_{\xi}=\left\{z \in \mathbb{C}^{n}:(z, \xi)=\sum_{k=1}^{n} z_{k} \overline{\xi_{k}}=0\right\}
$$

the complex hyperplane through the origin, perpendicular to $\xi$. Under the standard mapping from $\mathbb{C}^{n}$ to $\mathbb{R}^{2 n}$ the hyperplane $H_{\xi}$ turns into a $(2 n-2)$-dimensional subspace of $\mathbb{R}^{2 n}$.

Denote by $C_{c}\left(S^{2 n-1}\right)$ the space of $R_{\theta}$-invariant continuous functions, i.e. continuous real-valued functions $f$ on the unit sphere $S^{2 n-1}$ in $\mathbb{R}^{2 n}$ satisfying $f(\xi)=f\left(R_{\theta}(\xi)\right)$ for all $\xi \in S^{2 n-1}$ and all $\theta \in[0,2 \pi]$. The complex spherical Radon transform is an operator $\mathcal{R}_{c}: C_{c}\left(S^{2 n-1}\right) \rightarrow$ $C_{c}\left(S^{2 n-1}\right)$ defined by

$$
\mathcal{R}_{c} f(\xi)=\int_{S^{2 n-1} \cap H_{\xi}} f(x) d x .
$$

We say that a finite Borel measure $\mu$ on $S^{2 n-1}$ is $R_{\theta}$-invariant if for any continuous function $f$ on $S^{2 n-1}$ and any $\theta \in[0,2 \pi]$,

$$
\int_{S^{2 n-1}} f(x) d \mu(x)=\int_{S^{2 n-1}} f\left(R_{\theta} x\right) d \mu(x) .
$$


The complex spherical Radon transform of an $R_{\theta}$-invariant measure $\mu$ is defined as a functional $\mathcal{R}_{c} \mu$ on the space $C_{c}\left(S^{2 n-1}\right)$ acting by

$$
\left(\mathcal{R}_{c} \mu, f\right)=\int_{S^{2 n-1}} \mathcal{R}_{c} f(x) d \mu(x) .
$$

Complex intersection bodies were introduced and studied in [KPZ]. An origin symmetric complex star body $K$ in $\mathbb{R}^{2 n}$ is called a complex intersection body if there exists a finite Borel $R_{\theta}$-invariant measure $\mu$ on $S^{2 n-1}$ so that $\|\cdot\|_{K}^{-2}$ and $\mathcal{R}_{c} \mu$ are equal as functionals on $C_{c}\left(S^{2 n-1}\right)$, i.e. for any $f \in C_{c}\left(S^{2 n-1}\right)$

$$
\int_{S^{2 n-1}}\|x\|_{K}^{-2} f(x) d x=\int_{S^{2 n-1}} \mathcal{R}_{c} f(\theta) d \mu(\theta) .
$$

Theorem 3. Suppose that $K$ is a complex intersection body in $\mathbb{R}^{2 n}, f$ is an even continuous $R_{\theta}$-invariant function on $K, f \geq 1$ everywhere on $K$, and $\varepsilon>0$. If

$$
\int_{K \cap H_{\xi}} f \leq\left|K \cap H_{\xi}\right|+\varepsilon, \quad \forall \xi \in S^{2 n-1},
$$

then

$$
\int_{K} f \leq|K|+\frac{n}{n-1} d_{n}|K|^{1 / n} \varepsilon
$$

where $d_{n}=\left|B_{2}^{2 n}\right|^{\frac{n-1}{n}} /\left|B_{2}^{2 n-2}\right|<1$.

Proof : Use the polar formulas (4) and (5) to write the condition (15) in terms of the complex spherical Radon transform: for all $\xi \in S^{2 n-1}$

$$
\mathcal{R}_{c}\left(\int_{0}^{\|\cdot\|_{K}^{-1}} r^{2 n-3} f(r \cdot) d r\right)(\xi) \leq \frac{1}{2 n-2} \mathcal{R}_{c}\left(\|\cdot\|_{K}^{-2 n+2}\right)(\xi)+\varepsilon .
$$

Let $\mu$ be the measure on $S^{2 n-1}$ corresponding to $K$ by (14). Integrate the latter inequality over $S^{2 n-1}$ with the measure $\mu$ and use (14):

$$
\begin{gathered}
\int_{S^{2 n-1}}\|\theta\|_{K}^{-2}\left(\int_{0}^{\|\theta\|_{K}^{-1}} r^{2 n-3} f(r \theta) d r\right) d \theta \\
\leq \frac{1}{2 n-2} \int_{S^{2 n-1}}\|\theta\|_{K}^{-2 n} d \theta+\varepsilon \int_{S^{2 n-1}} d \mu(\xi) \\
=\frac{n}{n-1}|K|+\varepsilon \int_{S^{2 n-1}} d \mu(\xi) .
\end{gathered}
$$


Recall (2), (3) and the assumption that $f \geq 1$. We estimate the integral in the left-hand side of (17) as follows:

$$
\begin{gathered}
\int_{S^{2 n-1}}\|\theta\|_{K}^{-2}\left(\int_{0}^{\|\theta\|_{K}^{-1}} r^{2 n-3} f(r \theta) d r\right) d \theta \\
=\int_{S^{2 n-1}}\left(\int_{0}^{\|\theta\|_{K}^{-1}} r^{2 n-1} f(r \theta) d r\right) d \theta \\
+\int_{S^{2 n-1}}\left(\int_{0}^{\|\theta\|_{K}^{-1}}\left(\|\theta\|_{K}^{-2}-r^{2}\right) r^{2 n-3} f(r \theta) d r\right) d \theta \\
\geq \int_{K} f+\int_{S^{2 n-1}}\left(\int_{0}^{\|\theta\|_{K}^{-1}}\left(\|\theta\|_{K}^{-2}-r^{2}\right) r^{2 n-3} d r\right) d \theta \\
=\int_{K} f+\frac{1}{2(n-1) n} \int_{S^{2 n-1}}\|\theta\|_{K}^{-2 n} d \theta=\int_{K} f+\frac{1}{n-1}|K| .
\end{gathered}
$$

Let us estimate the second term in the right-hand side of (17) by adding the complex spherical Radon transform of the unit constant function under the integral $\left(\mathcal{R}_{c} 1(\xi)=\left|S^{2 n-3}\right|\right.$ for every $\left.\xi \in S^{2 n-1}\right)$, using again (14) and then applying Hölder's inequality:

$$
\begin{gathered}
\varepsilon \int_{S^{2 n-1}} d \mu(\xi)=\frac{\varepsilon}{\left|S^{2 n-3}\right|} \int_{S^{2 n-1}} \mathcal{R}_{c} 1(\xi) d \mu(\xi) \\
=\frac{\varepsilon}{\left|S^{2 n-3}\right|} \int_{S^{2 n-1}}\|\theta\|_{K}^{-2} d \theta \\
\leq \frac{\varepsilon}{\left|S^{2 n-3}\right|}\left|S^{2 n-1}\right|^{\frac{n-1}{n}}\left(\int_{S^{2 n-1}}\|\theta\|_{K}^{-2 n} d \theta\right)^{\frac{1}{n}} \\
=\frac{\varepsilon}{\left|S^{2 n-3}\right|}\left|S^{2 n-1}\right|^{\frac{n-1}{n}}(2 n)^{1 / n}|K|^{1 / n}=\frac{n}{n-1} d_{n}|K|^{1 / n} \varepsilon .
\end{gathered}
$$

In the last step we used $\left|S^{2 n-1}\right|=2 n\left|B_{2}^{2 n}\right|$. Combining (17),(18),(19) we get

$$
\int_{K} f+\frac{1}{n-1}|K| \leq \frac{n}{n-1}|K|+\frac{n}{n-1} d_{n}|K|^{1 / n} \varepsilon .
$$

It was proved in [KPZ] that if $K$ is a complex intersection body in $\mathbb{R}^{2 n}$ and $\gamma$ is an arbitrary measure on $\mathbb{R}^{2 n}$ with even continuous density, then

$$
\gamma(K) \leq \frac{n}{n-1} d_{n} \max _{\xi \in S^{2 n-1}} \gamma\left(K \cap H_{\xi}\right)|K|^{\frac{1}{n}} .
$$


We remove the condition that $K$ is a complex intersection body at the expense of an extra constant.

We use a result from [KPZ, Theorem 2] that a complex star body is a complex intersection body if and only if $\|\cdot\|_{K}^{-2}$ is a positive definite distribution, i.e. its Fourier transform in the sense of distributions assumes non-negative values on non-negative test functions. We refer the reader to [K1, KPZ] for details.

Theorem 4. Suppose that $L$ is an origin-symmetric complex convex body in $\mathbb{R}^{2 n}$ and $\gamma$ is an arbitrary measure on $\mathbb{R}^{2 n}$ with even continuous density $g$, then

$$
\gamma(L) \leq 2 n \frac{n}{n-1} d_{n} \max _{\xi \in S^{2 n-1}} \gamma\left(L \cap H_{\xi}\right)|L|^{\frac{1}{n}} .
$$

Proof : By John's theorem $[\mathrm{J}]$, there exists an origin-symmetric ellipsoid $K$ such that

$$
\frac{1}{\sqrt{2 n}} K \subset L \subset K
$$

Construct a new body $K_{c}$ by

$$
\|x\|_{K_{c}}^{-2}=\frac{1}{2 \pi} \int_{0}^{2 \pi}\left\|R_{\theta} x\right\|_{K}^{-2} d \theta .
$$

Clearly, $K_{c}$ is $R_{\theta}$-invariant, so it is a complex star body. For every $\theta \in[0,2 \pi]$ the distribution $\left\|R_{\theta} x\right\|_{K}^{-2}$ is positive definite, because this is a linear transformation of the Euclidean norm. So $\|x\|_{K_{c}}^{-2}$ is also a positive definite distribution, and, by [KPZ, Theorem 2], $K_{c}$ is a complex intersection body. Since $\frac{1}{\sqrt{2 n}} K \subset L \subset K$ and $L$ is $R_{\theta}$-invariant as a complex convex body, we have

$$
\frac{1}{\sqrt{2 n}} R_{\theta} K \subset L \subset R_{\theta} K, \quad \forall \theta \in[0,2 \pi]
$$

SO

$$
\frac{1}{\sqrt{2 n}} K_{c} \subset L \subset K_{c}
$$

Let $f=\chi_{K_{c}}+g \chi_{L}$, where $\chi_{K_{c}}, \chi_{L}$ are the indicator functions of $K_{c}$ and $L$. Clearly, $f$ is $R_{\theta}$-invariant and $f \geq 1$ everywhere on $K$. Put

$$
\varepsilon=\max _{\xi \in S^{2 n-1}}\left(\int_{K_{c} \cap H_{\xi}} f-\left|K_{c} \cap H_{\xi}\right|\right)=\max _{\xi \in S^{2 n-1}} \int_{L \cap H_{\xi}} g
$$


and apply Theorem 3 to $f, K_{c}, \varepsilon$ (the function $f$ is not necessarily continuous on $K_{c}$, but the result holds by a simple approximation argument). We get

$$
\begin{gathered}
\mu(L)=\int_{L} g=\int_{K_{c}} f-\left|K_{c}\right| \\
\leq \frac{n}{n-1} d_{n}\left|K_{c}\right|^{1 / n} \max _{\xi \in S^{2 n-1}} \int_{L \cap H_{\xi}} g \\
\leq 2 n \frac{n}{n-1} d_{n}|L|^{1 / n} \max _{\xi \in S^{2 n-1}} \mu\left(L \cap H_{\xi}\right),
\end{gathered}
$$

because $\left|K_{c}\right|^{1 / n} \leq 2 n|L|^{1 / n}$.

Theorem 4 shows that if bodies have additional symmetries then maximum in the slicing inequality can be taken over a rather small set of subspaces.

Acknowledgement. I wish to thank the US National Science Foundation for support through grant DMS-1265155.

\section{REFERENCES}

[Ba] K. Ball, Logarithmically concave functions and sections of convex sets in $R^{n}$, Studia Math. 88 (1988), 69-84.

[Bo1] J. Bourgain, On high-dimensional maximal functions associated to convex bodies, Amer. J. Math. 108 (1986), 1467-1476.

[Bo2] J. Bourgain, Geometry of Banach spaces and harmonic analysis, Proceedings of the International Congress of Mathematicians (Berkeley, Calif., 1986), Amer. Math. Soc., Providence, RI, 1987, 871-878.

[Bo3] J. Bourgain, On the distribution of polynomials on high-dimensional convex sets, Geometric aspects of functional analysis, Israel seminar (1989-90), Lecture Notes in Math. 1469 Springer, Berlin, 1991, 127-137.

[BGVV] S. Brazitikos, A. Giannopoulos, P. Valettas and B. Vritsiou, Geometry of isotropic log-concave measures, preprint.

[G] R. J. Gardner, Geometric tomography, Second edition, Cambridge University Press, Cambridge, 2006.

[GZ] E. Grinberg and Gaoyong Zhang, Convolutions, transforms and convex bodies, Proc. London Math. Soc. 78 (1999), 77-115.

[J] F. John, Extremum problems with inequalities as subsidiary conditions, Courant Anniversary Volume, Interscience, New York (1948), 187-204.

[Kl] B. Klartag, On convex perturbations with a bounded isotropic constant, Geom. Funct. Anal. 16 (2006), 1274-1290.

[K1] A. Koldobsky, Fourier analysis in convex geometry, Amer. Math. Soc., Providence RI, 2005.

[K2] A. Koldobsky, A hyperplane inequality for measures of convex bodies in $\mathbb{R}^{n}, n \leq 4$, Dicrete Comput. Geom. 47 (2012), 538-547. 
[K3] A. Koldobsky, $A \sqrt{n}$ estimate for measures of hyperplane sections of convex bodies, arXiv:1309.5271.

[KM] A. Koldobsky and Dan Ma, Stability and slicing inequalities for intersection bodies, Geom. Dedicata 162 (2013), 325-335

[KPZ] A. Koldobsky, G. Paouris and M. Zymonopoulou, Complex intersection bodies, J. London Math. Soc. 2013; doi: 10.1112/jlms/jdt014

[L] E. Lutwak, Intersection bodies and dual mixed volumes, Adv. Math. 71 (1988), $232-261$.

[Mi] E. Milman, Generalized intersection bodies. J. Funct. Anal. 240 (2) (2006), $530-567$.

[MP] V. Milman and A. Pajor, Isotropic position and inertia ellipsoids and zonoids of the unit ball of a normed n-dimensional space, in: Geometric Aspects of Functional Analysis, ed. by J. Lindenstrauss and V. Milman, Lecture Notes in Mathematics 1376, Springer, Heidelberg, 1989, pp. 64-104.

[Z] Gaoyong Zhang, Section of convex bodies, Amer. J. Math. 118 (1996), 319-340.

Department of Mathematics, University of Missouri, Columbia, MO 65211

E-mail address: koldobskiya@missouri.edu 